\title{
Bessel Function and Damped Simple Harmonic Motion
}

\author{
Masoud Asadi-Zeydabadi \\ Department of Physics, University of Colorado Denver, Denver, USA \\ Email: Masoud.Asadi-Zeydabadi@ucdenver.edu
}

Received 5 February 2014; revised 5 March 2014; accepted 9 March 2014

Copyright (C) 2014 by author and Scientific Research Publishing Inc.

This work is licensed under the Creative Commons Attribution International License (CC BY). http://creativecommons.org/licenses/by/4.0/

(c) (i) Open Access

\begin{abstract}
A glance at Bessel functions shows they behave similar to the damped sinusoidal function. In this paper two physical examples (pendulum and spring-mass system with linearly increasing length and mass respectively) have been used as evidence for this observation. It is shown in this paper how Bessel functions can be approximated by the damped sinusoidal function. The numerical method that is introduced works very well in adiabatic condition (slow change) or in small time (independent variable) intervals. The results are also compared with the Lagrange polynomial.
\end{abstract}

\section{Keywords}

Bessel Function; Simple Harmonic Motion; Damped Sinusoidal Function; Lengthening Pendulum; Spring-Mass System; Lagrange Polynomial

\section{Introduction}

A brief view of the graphs of Bessel and sinusoidal functions shows they are very similar. Bessel functions look like damped sinusoidal functions. Sinusoidal functions are well known for all of us and we have seen the foot prints of them almost everywhere. We knew them from trigonometry but Bessel functions are new for college students and seem more complicated and the students get familiar with them usually in differential equation. Bessel and sinusoidal functions are solution of Bessel and harmonic differential equations. We know these differential equations belong to the family of Sturm-Liouville equation. Bessel and sinusoidal functions are orthogonal function and they appear in the solution of some partial differential equations. The type of orthogonal function that appears in the solution depends on the geometry, physics (the form of the differential equation) and the boundary conditions. In spite of the similarity between them still for the students dealing with Bessel function is more difficult than sinusoidal function.

The purpose of this paper is using a pedagogical method to show the similarity between them through two 
physical examples. Basically this is an attempt to understand the mathematics through physics. This method is based on the similarity between the form of Bessel and sinusoidal functions and their similarity has been interpreted by these examples. This paper is not aiming to discuss or prove fundamental similarities or differences between these two groups of functions. Basically our observation shows Bessel functions behave like sinusoidal functions with decreasing amplitude and varying period. In this paper two examples are given to understand the root of these behaviors. These examples are the lengthening pendulum and spring-mass system with variable mass. Both length and mass in the pendulum and the spring-mass system respectively increase linearly with time. Finally for these examples the results of the exact solution (Bessel function) are compared with the approximation method (damped sinusoidal function).

In addition to this pedagogical method (physical perspective of Bessel equation) the damped sinusoidal function is a good numerical approximation for Bessel function. It is compared with the Lagrange polynomial fitting; this method provides results better than the Lagrange polynomial fitting.

\section{The Lengthening Pendulum}

The lengthening pendulum which is known also as Lorentz's pendulum is similar to a simple pendulum with increasing length $\left(l=l_{0}+v t\right)$ at constant rate $(v>0)$, with initial length of $l_{0}$ [1]-[8]. Its equation of motion is

$$
\frac{\mathrm{d}^{2} \theta}{\mathrm{d} t^{2}}+\frac{2 v}{l} \frac{\mathrm{d} \theta}{\mathrm{d} t}+\frac{g}{l} \sin \theta=0
$$

where $\theta$ is the pendulum angle relative to the vertical axis and $g$ is gravitational acceleration. By changing variable from $t$ to $l$ and by using the small angle approximation (1) becomes:

$$
\frac{\mathrm{d}^{2} \theta}{\mathrm{d} l^{2}}+\frac{2}{l} \frac{\mathrm{d} \theta}{\mathrm{d} l}+\frac{g}{v^{2} l} \theta=0 .
$$

The solution of (2) is given by Bessel function as following

$$
\theta=A \frac{J_{1}(u)}{u}+B \frac{Y_{1}(u)}{u}
$$

where $u=\frac{2 \sqrt{g l}}{v}$. If $\theta=\theta_{0}$ and $\dot{\theta}=0$ at $t=0$ then the constants are given by $A=-\frac{\pi u_{0}^{2}}{2} \theta_{0} Y_{2}\left(u_{0}\right)$ and $B=\frac{\pi u_{0}^{2}}{2} \theta_{0} J_{2}\left(u_{0}\right)$ where $u_{0}$ is the initial value of $u$. If $l_{0}$ and $v$ are related to each other such that $u_{0}=\frac{2 \sqrt{g l_{0}}}{v}$ is a zero of $J_{2}\left(u_{0}\right)$ then the solution is

$$
\theta=C \frac{J_{1}(u)}{u}
$$

where $C=\frac{u_{0} \theta_{0}}{J_{1}\left(u_{0}\right)}$.

To understand the solution in terms of the sinusoidal function, Equation (1) for the small angle approximation can be written as

$$
\frac{\mathrm{d}^{2} \theta}{\mathrm{d} t^{2}}+2 \gamma(t) \frac{\mathrm{d} \theta}{\mathrm{d} t}+\omega_{0}^{2}(t) \theta=0
$$

where $\gamma(t)=\frac{v}{l}$ and $\omega_{0}^{2}(t)=\frac{g}{l}$. Notice that since $l$ is time dependent, $\gamma(t)$ and $\omega_{0}(t)$ are functions of time. If an adiabatic condition (slow change) is considered or if small time intervals are chosen then $\gamma(t)$ and $\omega_{0}(t)$ are almost constant and the equation of motion is similar to damped simple pendulum. Then its solution for under damped condition $\left(\gamma^{2}<\omega_{0}^{2}\right)$ is $\theta=\mathrm{e}^{-\gamma(t) t}\left[c_{1} \sin (\omega(t) t)+c_{2} \cos (\omega(t) t)\right]$ where frequency of motion is given by $\omega^{2}=\omega_{0}^{2}-\gamma^{2}$ and it is function of time. For small time interval $\Delta t=\left(t_{n}-t_{n-1}\right)$ the solution at 
$t_{n}$ can be written in terms of the conditions of the pendulum at $t_{n-1}$ : $\theta_{n}=\mathrm{e}^{-\gamma_{n-1} \Delta t}\left[c_{1, n-1} \sin \left(\omega_{n-1}(\Delta t)\right)+c_{2, n-1} \cos \left(\omega_{n-1}(\Delta t)\right)\right]$. This solution shows how Bessel functions can be related to the damped sinusoidal solution.

The equation of motion can also be written in terms of $u=\frac{2 \sqrt{g l}}{v}$ which is a dimensionless variable as following:

$$
\frac{\mathrm{d}^{2} \theta}{\mathrm{d} u^{2}}+\frac{3}{u} \frac{\mathrm{d} \theta}{\mathrm{d} u}+\theta=0
$$

In this case the damping coefficient is $\gamma(u)=\frac{3}{2 u}$. Again under adiabatic condition the solution at the neighborhood of $u_{n}$ is $\theta \cong A_{n} \mathrm{e}^{-\gamma_{n} u} \sin \left(\omega_{n} u+a_{n}\right)$, where $\gamma_{n}=\frac{3}{2 u_{n}}, \omega_{n}^{2}=1-\gamma_{n}^{2}$ and for $\gamma_{n} \ll \omega_{n}$ we have $\omega_{n} \cong 1$. Therefore for large $u$ the period approaches to $2 \pi$ like sinusoidal function. By comparing with the exact solution (Bessel function) at the neighborhood of $u_{n}$ we have: $\frac{J_{1}(u)}{u} \cong C_{n} \mathrm{e}^{-\gamma_{n} u} \sin \left(\omega_{n} u+\beta_{n}\right)$ where $C_{n}$ and $\beta_{n}$ are given by the condition of the problem at $u_{n}$. This is an observation based on the solution of the lengthening pendulum and it is not a mathematical proof and depends on two constant $\left(C_{n}\right.$ and $\left.\beta_{n}\right)$ that should be determined by the condition at $u_{n}$.

\section{Spring-Mass System with Linearly Increasing Mass}

In this case the mass is increased in steady rate: $m=m_{0}+\alpha t$ where $m_{0}$ is the initial mass and $\alpha>0$ is the rate of change of the mass. The same treatment as previous case has been used. The equation of motion is:

$$
\frac{\mathrm{d}^{2} x}{\mathrm{~d} t^{2}}+\frac{\alpha}{m} \frac{\mathrm{d} x}{\mathrm{~d} t}+\frac{k}{m} x=0
$$

where $k$ is the spring constant. By changing variable from $t$ to $m$ in (7) we have

$$
\frac{\mathrm{d}^{2} x}{\mathrm{~d} m^{2}}+\frac{1}{m} \frac{\mathrm{d} x}{\mathrm{~d} m}+\frac{k}{\alpha^{2} m} x=0 .
$$

The solution of (8) is given by Bessel function as following:

$$
x=A J_{0}(u)+B Y_{0}(u)
$$

where $u=\frac{2 \sqrt{\mathrm{km}}}{\alpha}$. The initial conditions of $x(0)=x_{0}$ and $\dot{x}(0)=0$ give $A=-\frac{\pi u_{0}}{2} x_{0} Y_{1}\left(u_{0}\right)$ and $B=\frac{\pi u_{0}}{2} x_{0} J_{1}\left(u_{0}\right)$ where $u_{0}$ is the value of $u$ at $t=0$. If $m_{0}$ and $\alpha$ are adjusted such that $u_{0}=\frac{2 \sqrt{k m_{0}}}{\alpha}$ is a zero of $J_{1}\left(u_{0}\right)$ then the solution is

$$
x=C J_{0}(u)
$$

where $C=\frac{x_{0}}{J_{0}\left(u_{0}\right)}$. Equation (7) can be written as

$$
\frac{\mathrm{d}^{2} x}{\mathrm{~d} t^{2}}+2 \gamma(t) \frac{\mathrm{d} x}{\mathrm{~d} t}+\omega_{0}^{2}(t) x=0
$$

where $\gamma(t)=\frac{\alpha}{2 m}$ and $\omega_{0}^{2}(t)=\frac{k}{m}$. Since $m$ is time dependent, again $\gamma(t)$ and $\omega_{0}(t)$ are functions of time. The same as the pendulum case for adiabatic condition or for the small time interval $\gamma(t)$ and $\omega_{0}(t)$ 
are almost constant then the equation of motion is similar to damped harmonic motion. Then its solution for under damped condition $\left(\gamma^{2}<\omega_{0}^{2}\right)$ is $\theta=\mathrm{e}^{-\gamma(t) t}\left[c_{1} \sin (\omega(t) t)+c_{2} \cos (\omega(t) t)\right]$ where angular frequency of the motion is $\omega^{2}=\omega_{0}^{2}-\gamma^{2}$ and it is function of time.

The equation of motion in terms of $u=\frac{2 \sqrt{\mathrm{km}}}{\alpha}$ which is dimensionless is

$$
\frac{\mathrm{d}^{2} x}{\mathrm{~d} u^{2}}+\frac{1}{u} \frac{\mathrm{d} x}{\mathrm{~d} u}+x=0
$$

In this case the damping coefficient is $\gamma(u)=\frac{1}{2 u}$. Again under adiabatic condition the solution at the neighborhood of $u_{n}$ is $x \cong A_{n} \mathrm{e}^{-\gamma_{n} u} \sin \left(\omega_{n} u+a_{n}\right)$, where $\gamma_{n}=\frac{1}{2 u_{n}}, \omega_{n}^{2}=1-\gamma_{n}^{2}$ and for $\gamma_{n} \ll \omega_{n}$ we have $\omega_{n} \cong 1$. For large $u$ the period approaches to $2 \pi$ like sinusoidal function. By comparing with the exact solution (Bessel function) at the neighborhood of $u_{n}$ we have $J_{0}(u) \cong C_{n} \mathrm{e}^{-\gamma_{n} u} \sin \left(\omega_{n} u+\beta_{n}\right)$ where $C_{n}$ and $\beta_{n}$ are given by the conditions of the system at $u_{n}$. This is physical evidence based on observation and it is not a mathematical proof.

\section{The Quadratic Lagrange Polynomial for Numerical Comparison}

In general the damped sinusoidal function provides a good approximation for Bessel functions. It can be compared with the quadratic Lagrange polynomial fitting which is given by [9] [10]

$$
x(u)=\sum_{i=1}^{N}\left(\prod_{\substack{1 \leq j \leq N \\ j \neq i}} \frac{u-u_{j}}{u_{i}-u_{j}}\right) x_{i}
$$

For the quadratic case $N=3$ and three points, $\left(u_{1}, x_{1}\right),\left(u_{2}, x_{2}\right)$ and $\left(u_{3}, x_{3}\right)$, are needed.

\section{Results and Discussion}

In this section some results are shown for both cases. The numerical values are used are not based on any physical reason and they are used just for comparison of these two methods.

In both of these problems there are two independent variables $((\theta, l)$ for pendulum and $(x, m)$ for spring-mass system). Change in the momentum is due to both variables. Appearance of the first derivatives ( $\dot{\theta}$ and $\dot{x}$ for pendulum and spring-mass system respectively) make them different from the simple harmonic motion. This is because of the change of the momentum due to the second independent variable ( $l$ for the pendulum and $m$ for the spring-mass system). Mathematically this first derivative plays the role of the damping term that causes the amplitude decay. In reality there is no drag force like air resistance in these two cases but they are not conservative system their energies depend on time [1]. The total mechanical energy is the sum of the kinetic and the potential energy and for the pendulum case the energy density (energy per unit mass) is $\frac{E}{m}=\frac{1}{2}\left(i^{2}+l^{2} \dot{\theta}^{2}\right)-g l \cos \theta \cong \frac{1}{2}\left(i^{2}+l^{2} \dot{\theta}^{2}\right)-g l\left(1-\theta^{2} / 2\right)$. By substitution of (4) into this expression the energy density is given by $\frac{E}{m}=\frac{1}{2} v^{2}-g l+\frac{C^{2}}{8}\left(\left[J_{1}^{2}(u)+J_{2}^{2}(u)\right]\right) v^{2}$. The last (Bessel's) term is proportional to $v^{2}$ (like kinetic energy). This term decays down and the energy density approaches to $\frac{1}{2} v^{2}-g l$. Since $l$ increases with time the energy decreases. The power density is given by $\frac{p}{m}=\frac{1}{m} \frac{\mathrm{d} E}{\mathrm{~d} t}=-g v+\frac{v^{2} C}{8} \sqrt{\frac{g}{l}}\left[J_{1}(u) J_{0}(u)-J_{2}(u) J_{3}(u)\right]$. Again the Bessel's term decays and the power density converges to $-g v=-F_{T} v<0$ where $F_{T}$ is the string tension at this limit (at large value of time). Therefore the exact solution shows the energy of the system deceases as we expected from the approximation method 
based on the damped oscillation. Figure 1 shows the energy density and power density for the case of $v=$ $0.2048 \mathrm{~m} / \mathrm{s}$. It shows power density is negative and it decreases with time and approaches to $-g v=-2.007$ $\mathrm{W} / \mathrm{kg}$.

The amplitude decays as $\mathrm{e}^{-\gamma_{n} u}$ but $\gamma_{n}$ is inversely proportional to $u_{n}$ therefore the rate of the change of the amplitude decreases. The angular frequency $\omega_{n}=\sqrt{1-\gamma_{n}^{2}}$ increases as $u_{n}$ increases and approaches to 1 , $\left(\lim _{u_{n} \rightarrow \infty} \omega_{n}=1\right)$, therefore the period is inversely related to $u$ (it decreases as $u$ increases). This means for large $u$ the amplitude and frequency converge to almost constant values or the solution behaves like simple harmonic motion. We know $u$ by itself is function of time $\left(u=\frac{2 \sqrt{g l}}{v}\right.$ and $\left.u=\frac{2 \sqrt{\mathrm{km}}}{\alpha}\right)$ and it increases by time and consequently the period increases by time. This can be clarified by giving the frequencies in terms of ( $l$ and $\mathrm{m}$ ) which are functions of time. The frequencies in terms of $t$ for the pendulum and the spring-mass system are $\omega(t)=\sqrt{\frac{g}{l}-\frac{v^{2}}{l^{2}}}$ and $\omega(t)=\sqrt{\frac{k}{m}-\frac{\alpha^{2}}{4 m^{2}}}$ respectively. For large value of time (large $l$ and $m$ ) they approach to $\sqrt{\frac{g}{l}}$ and $\sqrt{\frac{k}{m}}$. That means they get smaller and the periods become larger as time increases. Figure 2 shows the results of two methods. For these results the small time intervals (less than the local period) have been used. This is a good criterion for the time step size in adaptive numerical method. Figure 2 shows the approximation method (damped sinusoidal method) agrees very well with the exact solution (Bessel function) for small time intervals. One can observe from this figure the period increases with time.

Suppose the pendulum problem has been solved exactly for some initial conditions. This solution is given in (4) by Bessel functions and at a given $u_{n}$ the exact conditions of the motion, $\left(\theta_{n}\right.$ and $\left.\dot{\theta}_{n}\right)$, can be found and substituted into, $\theta \cong A_{n} \mathrm{e}^{-\gamma_{n} u} \sin \left(\omega_{n} u+a_{n}\right)$, to find $A_{n}$ and $a_{n}$. Figure 3 shows this solution for $u_{0}=5.13562$ (the first zero of $J_{2}(u)$ ) with initial conditions of $\theta_{0}=10^{\circ}$ and $\dot{\theta}_{0}=0$. The solution corresponding to $u_{n}=100$ has been shown by a dot on the graph. At this point the exact and the approximation solutions should
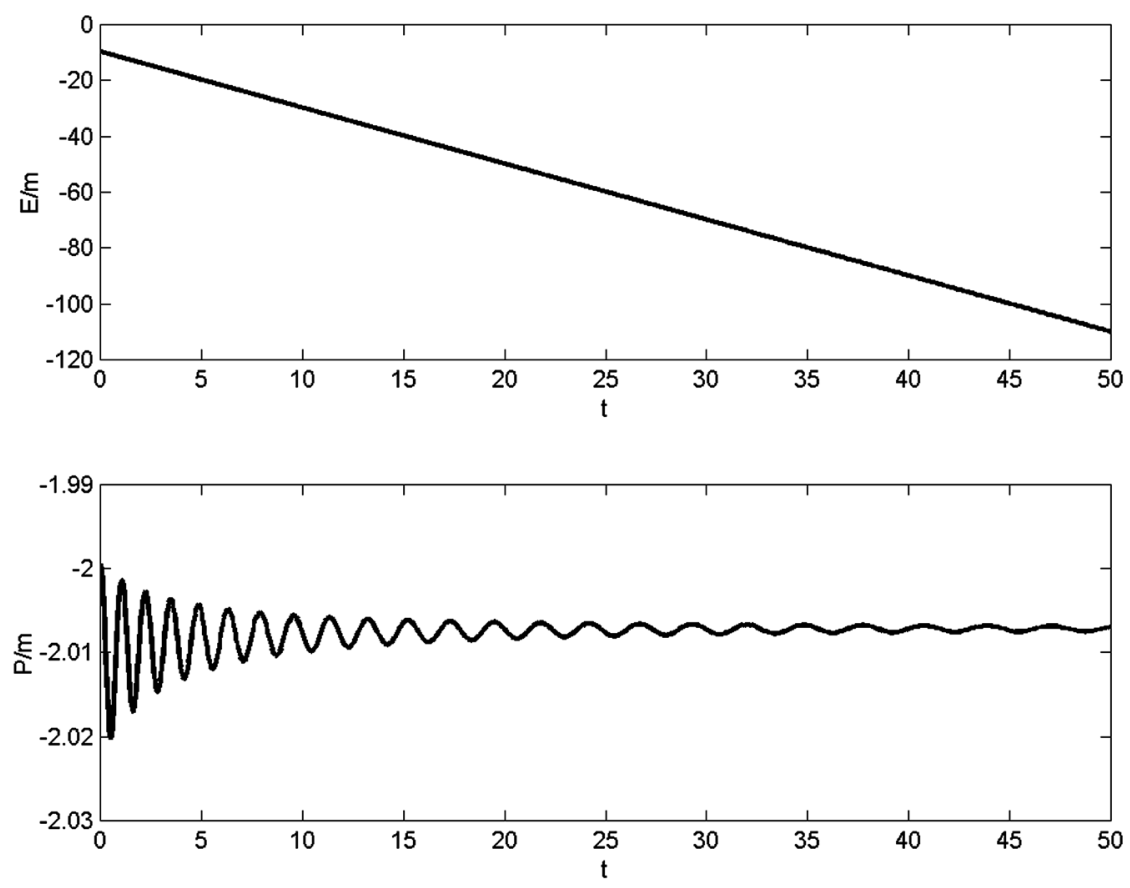

Figure 1. Energy density and power density for the lengthening pendulum: $u_{0}=30.571$ $v=0.2048 \mathrm{~m} / \mathrm{s}, \theta_{0}=5^{\circ}$ and $\dot{\theta}_{0}=0$. 


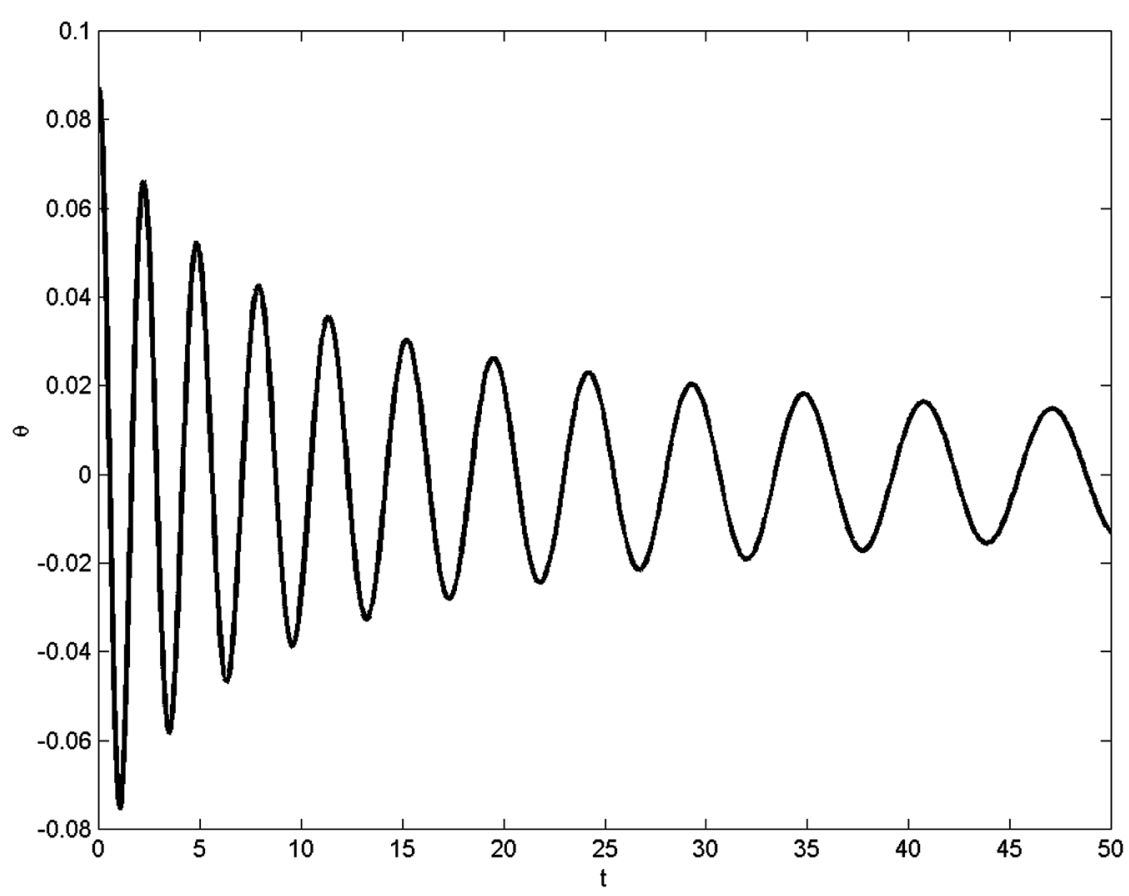

Figure 2. The results of exact (Bessel) and the approximation (damped sinusoidal) solution for the lengthening pendulum $\left(u_{0}=30.571, v=0.2048 \mathrm{~m} / \mathrm{s}, \theta_{0}=5^{\circ}\right.$ and $\left.\dot{\theta}_{0}=0\right)$. Since the small time interval has been used the results of both methods are quite agree with each other (no differences can be seen).
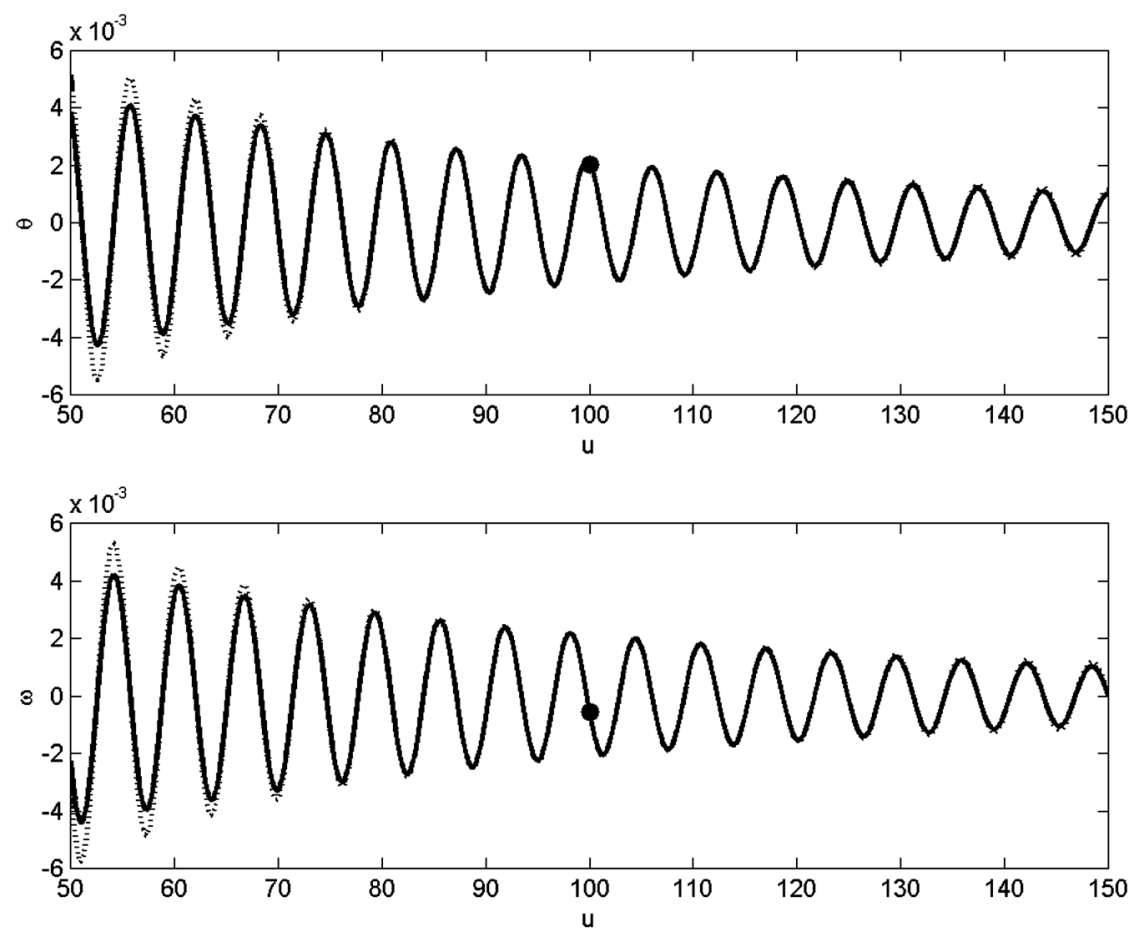

Figure 3. The results of the exact solution, Bessel function (solid line), and the approximation method, damped sinusoidal solution (dot line), for the lengthening pendulum: $l_{0}=$ $1 \mathrm{~m}, u_{0}=5.13562, \theta_{0}=10^{\circ}, \dot{\theta}_{0}=0, u_{n}=100, \theta_{n}=0.002 \mathrm{rad}$ and $\dot{\theta}_{n}=-5.6811 \times 10^{-4}$ $\mathrm{rad} / \mathrm{s}$. The solution corresponding to $u_{n}=100$ has been shown by a dot on the graph. 
match with each other through the condition at $u_{n}$ (i.e. $\theta_{n}$ and $\dot{\theta}_{n}$ ). Using these conditions from the exact solution and impose them on the approximation (damped sinusoidal) method gives us the approximation solution at the neighborhood of this point. Figure 3 shows the results agree with each other particularly near this point. One can see the deviation of the approximation method from the exact solution away from this point especially for small $u$. In this case we are not using small time interval and the figure shows the results over several periods.

Figure 4 shows the same results as Figure 3 but for the spring-mass system, in this case the exact solution is given in terms of $J_{0}(u)$. Again the results of the exact solution and the approximation method are quite similar. The deviation of these two methods at small $u$ is shown in Figure 5. Again we can observe for $u>u_{n}$ the results are matched very well.

In the Lagrange polynomial fitting three points are needed but for the damped sinusoidal function only the value of Bessel function and its derivative at a point are needed. This is a big advantage of this method compare to the Lagrange polynomial fitting. The Bessel function is oscillatory therefore the order of polynomial in a large interval should be higher and more points are needed. Figure 6 shows the comparison of these two techniques with the exact Bessel function.

The results from Figure 6 shows the damped sinusoidal function is a better approximation compare to the Lagrange polynomial fitting. It is obvious for the larger interval the quadratic polynomial is not a reasonable approximation and higher order polynomial with more points are needed. The results of this paper shows in long interval the damped sinusoidal is good numerical approximation.

\section{Conclusions}

The graphs of Bessel functions are similar to the damped sinusoidal solution. In this paper this observation has been investigated by two physical examples. The Bessel's equation has been compared with the equation of the damped simple harmonic motion. The solutions of these methods are compared at the neighborhood of an arbitrary point. The results are shown the approximation method works very well particularly when $u$ is large (i.e. for large value of independent variable for example time in this paper for two examples). The damped sinusoidal
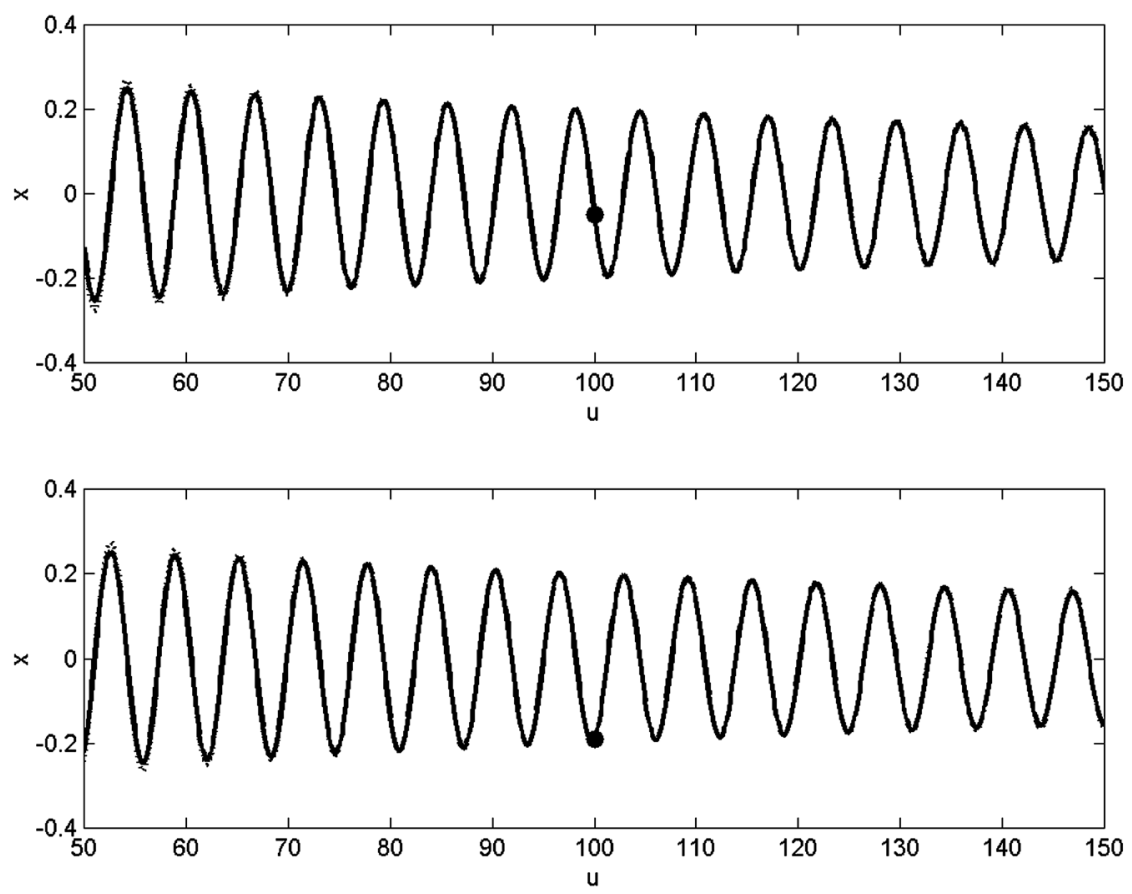

Figure 4. The results of the exact solution (Bessel function, solid line) and the approximation method (damped sinusoidal solution, dot line) for the mass-spring system: $m_{0}=1$ $\mathrm{kg}, u_{0}=3.832, x_{0}=1 \mathrm{~m}, u_{n}=100, x_{n}=-0.0496 \mathrm{~m}$ and $\dot{x}_{n}=-0.1915 \mathrm{~m} / \mathrm{s}$. The solution corresponding to $u_{n}=100$ has been shown by a dot on the graph. 

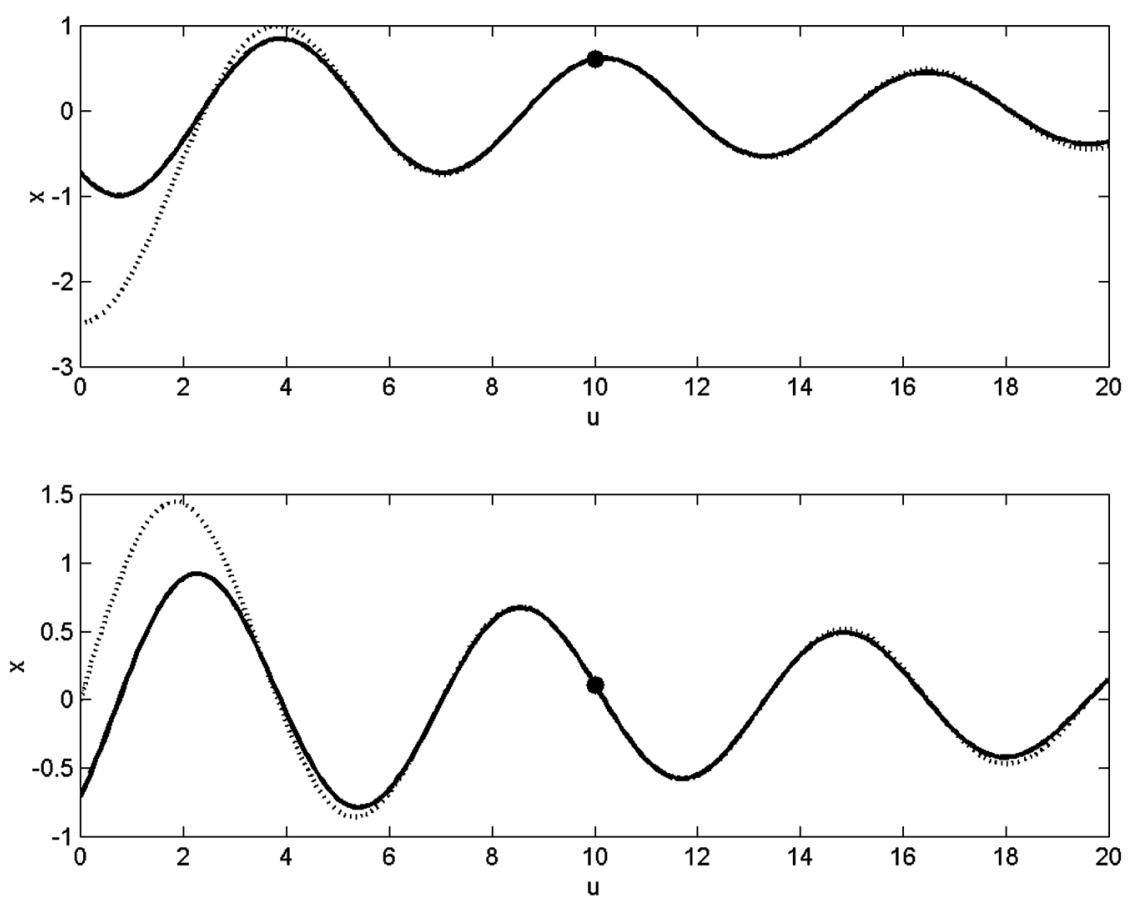

Figure 5. The results of the exact solution (Bessel function, solid line) and the approximation (damped sinusoidal, dot line) method for the mass-spring system: $m_{0}=1 \mathrm{~kg}, u_{0}=$ 3.832, $x_{0}=1 \mathrm{~m}, u_{n}=10, x_{n}=0.6106 \mathrm{~m}$ and $\dot{x}_{n}=0.1079 \mathrm{~m} / \mathrm{s}$. The solution corresponding to $u_{n}=10$, has been shown by a dot on the graph.

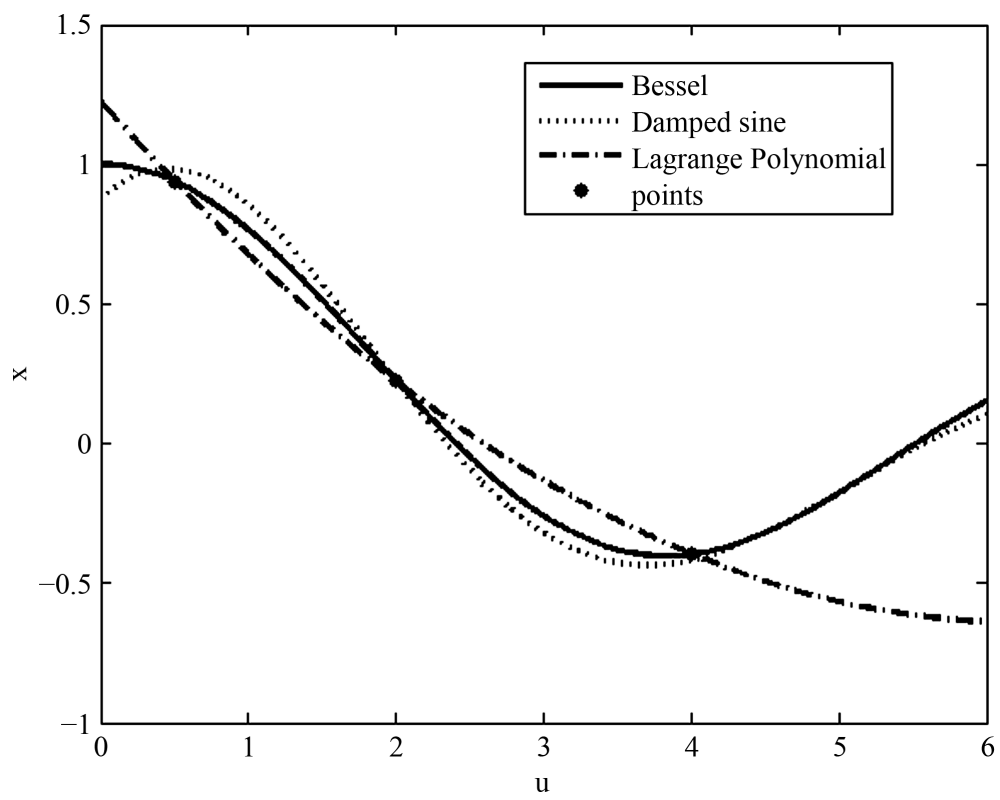

Figure 6. Bessel function, $x=J_{0}(u)$, the damped sinusoidal function, and the quadratic Lagrange polynomial. The given points for Bessel function, $J_{0}(0.5)$ $=0.9385, J_{0}(2.0)=0.2239$ and $J_{0}(4.0)=-0.3971$, are shown in figure.

function not only is a good way to interpret the property of the Bessel equation but also is a good numerical approximation. The comparison with the Lagrange polynomial shows the numerical advantage of the damped sinusoidal function. 
In this paper we use two physical examples but this method can be generalized for any Bessel's equation. The general form of Bessel's equation is $\frac{\mathrm{d}^{2} y}{\mathrm{~d}^{2}}+(1 / u) \frac{\mathrm{d} y}{\mathrm{~d} u}+\left[1-\left(p^{2} / u^{2}\right)\right] y=0$. Again by using the appropriate initial value for $u$ the final solution can be given in terms of either $J_{p}(u)$ or $Y_{p}(u)$. The damped sinusoidal solution at the neighborhood of a given point $\left(u_{n}\right)$ is given by $\left(y \cong A_{n} \mathrm{e}^{-\gamma_{n} u} \sin \left(\omega_{n} u+a_{n}\right)\right)$ where $\gamma_{n}=\frac{1}{u_{n}}$ and $\omega_{n}=\left[1-\left(p^{2} / u^{2}\right)\right]-\gamma_{n} \cong 1-\left(p^{2} / u^{2}\right)$. The values of Bessel function and its derivative at $u_{n}$ are used to find $A_{n}$ and $a_{n}$. This method can be considered as a good perturbation method for the problems that are involved Bessel function. This method can improve numerical techniques for some particular equations. In the same way we can look at other functions like Legendre polynomial. The Legendre differential equation is given by $\left(1-u^{2}\right) \frac{\mathrm{d}^{2} y}{\mathrm{~d} u^{2}}-2 u \frac{\mathrm{d} y}{\mathrm{~d} u}+\ell(\ell+1) y=0$ with $-1 \leq u \leq 1$. We can write the differential equation in the form damping harmonic oscillation $\frac{\mathrm{d}^{2} y}{\mathrm{~d} u^{2}}-\gamma \frac{\mathrm{d} y}{\mathrm{~d} u}+\omega^{2} y=0$ with $\gamma=\frac{2 u}{\left(1-u^{2}\right)}$ and $\omega=\frac{\ell(\ell+1)}{\left(1-u^{2}\right)}$. In this case the solution is growing up and the $\omega$ increases by $\ell$. Notice that the polynomial is defined in region of $-1 \leq u \leq 1$.

\section{References}

[1] Werner, A. and Eliezer, J.C. (1969) The Lengthening Pendulum. Journal of Australian Mathematical Society, 9, 331336. http://dx.doi.org/10.1017/S1446788700007254

[2] Littlewood, J.E. (1963) Lorentz’s Pendulum Problem. Annals of Physics, 21, 233-249. http://dx.doi.org/10.1016/0003-4916(63)90107-6

[3] Littlewood, J.E. (1964) Adiabatic Invariance III. The Equation $x=-V_{x}(x, \omega)$. Annals of Physics, 29, 1-12. http://dx.doi.org/10.1016/0003-4916(64)90188-5

[4] Littlewood, J.E. (1964) Adiabatic Invariance IV: Note on a New Method for Lorentz's Pendulum Problem. Annals of Physics, 29, 13-18. http://dx.doi.org/10.1016/0003-4916(64)90189-7

[5] Littlewood, J.E. (1964) Adiabatic Invariance V. Multiple Periods. Annals of Physics, 30, 138-153. http://dx.doi.org/10.1016/0003-4916(64)90307-0

[6] Brearley, M.N. (1966) The Simple Pendulum with Uniformly Changing String Length. Proceedings of the Edinburgh Mathematical Society (Series 2), 15, 61-66.

[7] Sánchez-Soto, L.L. and Zoido, J. (2013) Variations on the Adiabatic Invariance: The Lorentz Pendulum. American Journal of Physics, 81, 57. http://dx.doi.org/10.1119/1.4763746

[8] Boas, M.L. (2006) Mathematical Methods in the Physical Science. 3rd Edition, Wiley, 598-599.

[9] Gil, A., Segura, J. and Temme, N. (2007) Numerical Methods for Special Functions. SIAM. http://dx.doi.org/10.1137/1.9780898717822

[10] Garcia, A.L. (2000) Method for Physics. 2nd Edition, Prentice-Hall, NJ. 